Revue française de la traduction

\title{
Quelle(s) traduction(s) pour le terme anglais greenwashing? Quelques observations croisées en terminologie
}

Pascaline Dury

\section{(2) OpenEdition \\ Journals}

\section{Édition électronique}

URL : http://journals.openedition.org/traduire/580

DOI : $10.4000 /$ traduire.580

ISSN : 2272-9992

Éditeur

Société française des traducteurs

\section{Édition imprimée}

Date de publication : 15 décembre 2013

Pagination : 26-35

ISSN : 0395-773X

\section{Référence électronique}

Pascaline Dury, «Quelle(s) traduction(s) pour le terme anglais greenwashing ? Quelques observations croisées en terminologie », Traduire [En ligne], 229 | 2013, mis en ligne le 15 décembre 2015, consulté le 02 mai 2019. URL : http://journals.openedition.org/traduire/580 ; DOI : 10.4000/traduire.580 


\section{Quelle(s) traduction(s) pour le terme anglais greenwashing? \\ Quelques observations croisées \\ en terminologie}

\section{Pascaline Dury}

L'article qui suit propose les résultats d'un travail d'exploration en corpus, mené avec un œil de terminologue, sur les traductions possibles en français du terme anglais greenwashing, un terme utilisé dans le domaine du développement durable, de la publicité, et du marketing. L'objectif ici n'est pas de faire des recommandations sur la meilleure traduction possible de ce terme en français, mais plutôt de présenter une analyse croisée de quelques aspects qui nous paraissent importants dans l'usage des équivalents de greenwashing en français, afin que le lecteur puisse disposer d'informations utiles et convergentes préalablement à la traduction. À cette fin, nous concentrons notre analyse sur trois points : un premier point relatif au nombre d'occurrences en corpus des équivalents de traduction possibles de greenwashing; un second point lié au sens de ces termes, qui détaillera notamment leurs préférences sémantiques, et enfin un dernier point plus strictement lié à l'usage.

Mais qu'est-ce que le greenwashing? Le dictionnaire Oxford définit le terme greenwash de la façon suivante : "disinformation dissiminated by an organization so as to present an environmentally responsible public image ". II s'agit, en d'autres mots, de l'exploitation inadéquate ou abusive de l'image de développement durable ou d'arguments écologiques par une entreprise ou une organisation dans le cadre de sa communication.

Un traducteur à la recherche d'une terminologie officielle en français pour traduire ce terme se heurterait immédiatement à un écueil important, car il existe en effet deux termes officiels équivalents de greenwashing: le premier, "écoblanchiment(1) ", est proposé par le grand dictionnaire terminologique (GDT), avec la définition suivante : " opération de relations publiques menée par une organisation, une entreprise pour masquer ses activités polluantes et tenter de présenter un caractère écoresponsable ". Le second, " verdissement d'image ", a été publié tout récemment (le 8 septembre 2013) au Journal officiel de la République française par la

(1) Le GDT précise qu'outre le terme "écoblanchiment ", les termes " mascarade écologique ", "blanchiment vert " et " verdissement d'image " sont possibles. De la même façon, la Commission générale de terminologie et de néologie note que l'on trouve aussi les termes " écoblanchiment " et " blanchiment écologique ". 
Commission générale de terminologie et de néologie, avec la définition suivante : " attribution abusive de qualités écologiques à un produit, à un service ou à une organisation ".

Afin d'explorer plus avant l'usage de ces deux équivalents officiels, nous avons compilé un corpus ad-hoc de 341542 mots, constitué de textes et de documents rédigés en français, collectés sur la toile dans les pages France, et qui ont pour sujet le greenwashing. Ce corpus est supposé représenter un échantillon de l'usage " commun " le plus large possible : pour cette raison, nous avons retenu des passages de blogs, des sites personnels, des articles d'associations et de mouvements écologistes, des articles de journaux, des publications universitaires, etc. Nous soulignons d'ailleurs que les résultats présentés dans cet article ne valent que pour le corpus utilisé et qu'ils ne pourraient pas servir pour une extrapolation à la langue générale dans son ensemble. Le corpus a été intégralement exploré grâce aux outils proposés par le logiciel ANTCONC(2).

\section{Quels équivalents de traduction pour greenwashing, avec quelles occurrences?}

L'exploration du corpus a d'abord consisté à repérer les équivalents de traduction possibles de greenwashing présents dans le corpus. Le tableau 1 ci-dessous présente ces équivalents de traduction et leurs occurrences respectives.

\begin{tabular}{|l|c|l|}
\hline \multicolumn{1}{|c|}{ Équivalent de traduction } & Nombre d'occurrences en corpus & \\
\hline "Greenwashing " & 340 & \\
\hline " Verdissement d'image " & 198 & Terme publié au Journal officiel \\
\hline "Ecoblanchiment " & 167 & Terme préconisé par le GDT \\
\hline "Blanchiment écologique " & 100 & \\
\hline "Blanchiment vert " & 98 & \\
\hline " Verdissage " & 73 & \\
\hline " Maquillage vert " & 55 & \\
\hline " Mascarade écologique " & 43 & \\
\hline " Désinformation verte " & 41 & \\
\hline "Badigeonnage vert " & 28 & \\
\hline
\end{tabular}

Tableau 1 : traductions possibles du terme greenwashing en français avec leurs occurrences respectives en corpus

Les résultats extraits du corpus montrent qu'il existe en réalité huit termes possibles, outre les deux termes officiels, pour traduire greenwashing en français. Parmi ces équivalents de

(2) Ce concordancier est disponible gratuitement en ligne à l'adresse suivante :

http://www.antlab.sci.waseda.ac.jp/software.html 
traduction, quatre (dont les deux termes officiels) apparaissent plus de 100 fois dans notre corpus. On note en outre la supériorité numérique écrasante du terme " greenwashing " utilisé tel quel en français, comme le montrent les deux contextes ci-dessous issus du corpus :

"L'utilisation de certains éléments du greenwashing, comme la couleur, peut avoir certains effets négatifs..."

"Le "greenwashing" concerne généralement des enseignes de grande distribution ou des marques mondiales...".

À l'inverse, le terme " badigeonnage vert " est assez peu présent dans le corpus, et une rapide vérification de sa fréquence d'usage sur la toile, à l'aide de Google, confirme ce résultat : alors que les termes "greenwashing " (en français) et "verdissement d'image " apparaissent respectivement 134000 et 98000 fois sur internet dans les pages France, le terme " badigeonnage vert " n'apparaît que 111 fois. Par conséquent, on peut peut-être considérer que d'un point de vue strictement numérique, le terme " badigeonnage vert " se trouve en dessous d'un seuil de fiabilité qui n'en fait pas un équivalent de traduction privilégié.

De la même façon, le terme " mascarade écologique " est assez peu fréquent (43 occurrences) dans notre corpus, constitué, nous le rappelons, de documents collectés sur les pages France d'internet. Une recherche d'occurrences sur Google corrobore ce résultat jusqu'à un certain point seulement : " mascarade écologique " apparaît 220 fois sur internet si l'on restreint les recherches aux pages France effectivement, mais il apparaît beaucoup plus fréquemment (1 430 fois) dans les pages en français en général. Cela s'explique sans doute par le fait que, après vérification, la grande majorité des sources dans lesquelles on trouve le terme sont québécoises ; " mascarade écologique " est d'ailleurs mentionné dans le GDT comme synonyme "d'écoblanchiment ". Le terme " mascarade écologique " qu'on peut alors peut-être considérer comme une variante diatopique de "greenwashing ", semble plus représentatif d'un usage en français québécois qu'en français hexagonal.

Malgré tout l'intérêt que représente l'information chiffrée sur les équivalents de traduction possibles de greenwashing, il nous semble qu'elle ne peut, à elle seule, constituer une aide fiable pour la traduction et qu'il faut y voir des tendances, plutôt que des règles strictes. D'abord, nous l'avons déjà souligné, parce que les observations de ce genre ne valent strictement que par rapport à un corpus déterminé, constitué par un certain type de textes ; ensuite parce que le développement durable est à la mode et que le concept du greenwashing est un concept qui suscite, depuis le début, l'intérêt des internautes : ceci n'est pas sans conséquence sur l'usage reflété par le web. En effet, en analysant les documents du corpus plus en détail, nous avons remarqué que nombre de sources issues de la toile, bien que d'origines et d'auteurs différents, copient en réalité tout ou partie du contenu présent dans d'autres sources internet et ne font que répéter les définitions et les exemples donnés par d'autres. Cela pose un problème par rapport à l'usage des termes étudiés, dans la mesure où internet, et à plus 
petite échelle, notre corpus(3) reflètent alors un usage répété, copié, donc amplifié et sans doute stéréotypé du terme, plutôt qu'un usage spontané, "individuel " et peut-être plus " authentique".

Enfin, même si l'information chiffrée sur les équivalents de traduction possibles renseigne sur le ou les équivalents les plus ou les moins usités, elle ne permet pas de faire un choix de traduction parmi les équivalents dont les fréquences sont relativement proches, comme c'est le cas ici pour quatre termes : "verdissement d'image " (198 occurrences), " écoblanchiment " (167 occurrences), " blanchiment écologique " (100 occurrences) et " blanchiment vert " (98 occurrences). II faut donc recourir à une analyse de type sémantique afin d'en apprendre plus sur le sens exact de ces termes et sur leur usage.

\section{Quelle(s) préférence(s) sémantique(s) pour quel(s) équivalent(s) de traduction ?}

Nous abordons ici la question sémantique d'un point de vue un peu particulier qui est celui des affinités électives et des préférences sémantiques, aussi appelées la prosodie sémantique. Le principe des préférences sémantiques (semantic preferences) a été développé, pour la langue générale, par Sinclair (1987), puis a été repris par d'autres, notamment Louw (1993), Stubbs (2001) et Partington (2004). La prosodie sémantique repose sur l'idée que certains termes peuvent montrer une préférence sémantique, ou des affinités électives, pour une famille de termes sémantiquement proches, avec lesquels ils sont associés, parfois en co-occurrence, selon une fréquence plus élevée que le simple hasard et que cette préférence sémantique conduit à les utiliser dans des contextes plutôt que d'autres. Selon Partington (145) : " semantic preference [is] the relation not between individual words, but between a lemma or word-form and a set of semantically related words ". Pour Stubbs (65) : " an item shows semantic preference when it co-occurs with a class of words which share some semantic feature".

Nous avons donc examiné de très près, dans notre corpus, la rencontre non-fortuite de deux mots ou plutôt d'un mot et d'une famille de mots sémantiquement liés, et recherché ainsi les préférences sémantiques des termes à l'étude pour repérer les points communs et les différences éventuelles qui orienteraient l'utilisation de tel ou tel équivalent de traduction.

(3) Nous avons procédé à un tri des documents constituant le corpus et enlevé, autant que possible, les sites pour lesquels les redondances étaient flagrantes, mais ce tri ayant été fait manuellement, le risque d'erreur reste important. 


\subsection{Une première famille de préférences sémantiques}

Les résultats obtenus suggèrent que les termes " greenwashing ", " désinformation verte ", " badigeonnage vert ", " maquillage vert " et " mascarade écologique " (nous les nommerons désormais, par souci de simplification, "la famille greenwashing ") partagent des préférences sémantiques identiques qui s'organisent autour de trois thèmes saillants :

1. L'accompagnateur de loin le plus spécifique est le terme " abus " (et les formes liées " abusive ", " abuser ") que l'on trouve accolé dans presque un cas sur deux aux termes de "la famille greenwashing ". En voici quelques exemples tirés du corpus :

"Le "greenwashing" ou l'exploitation abusive de l'image environnementale dans la communication des entreprises..."

"Le "greenwashing" abuse ou utilise à mauvais escient l'argument écologique "

"La désinformation verte désigne l'utilisation abusive et mensongère de l'argument écologique "

Outre l'abus, on note d'ailleurs, dans notre corpus, une tendance très marquée des termes de la "famille greenwashing " à se combiner avec des formes lexicales exprimant la tromperie, le mensonge et décrivant une pratique déloyale :

"La mascarade écologique est le fait de tromper les consommateurs à propos des pratiques environnementales d'une entreprise..."

"Le "greenwashing" induit le public en erreur en mettant en avant... "

"Le "badigeonnage vert" (ou "greenwashing") est une pratique déloyale qui sape la confiance des consommateurs. "

"Le greenwashing est une technique marketing mensongère pour donner une dimension écologique à une entreprise. "

"Pourtant, aucune entreprise, qu'elle soit petite ou grande n'est à l'abri de ce mal insidieux "

"Ce qu'on appelle le greenwashing est la dérive la plus malsaine de notre environnement publicitaire. "

2. Les termes de la "famille greenwashing " se rapportent également avec une fréquence très élevée à l'évocation de la méfiance vis-à-vis de ces pratiques abusives et à la nécessité de les éviter, voire de les combattre. Le terme " prévenir " semble être, en l'occurrence, un partenaire privilégié :

" II faut à tout prix combattre la mascarade écologique et lutter contre les campagnes publicitaires contenant des affirmations écologiques non justifiées" 
"... pour nous éviter de tomber tous dans le piège du "greenwashing" "

"Prévenir le "greenwashing" est un défi majeur "

"La prévention du "greenwashing" suppose d'identifier les pratiques trompeuses "

"...qui ont pour but de prévenir la mascarade écologique "

3. Enfin, un autre type de combinaison préférentielle de la " famille greenwashing " semble se faire autour des termes à connotation juridique, exprimant notamment l'idée d'infraction, de délit.

"La mascarade écologique est un danger réel d'atteinte à la réputation "

" ...jusqu'à $99 \%$ sont coupables d'une manière ou d'une autre de maquillage vert "

"...pour les entreprises qui en sont coupables, ce procédé de vente fallacieux peut prendre des formes multiples"

"...sont suspectées de pratiquer une forme ou l'autre de mascarade écologique "

" ...en effet, tombent sous le coup de "greenwashing" les publicités qui utilisent le thème de la nature..."

"...est un cas avéré de greenwashing publicitaire "

\subsection{Une préférence sémantique pour les termes qui évoquent l'argent}

Les résultats extraits du corpus montrent également que trois variantes, "blanchiment vert ", "blanchiment écologique ", et " écoblanchiment " partagent les mêmes ressemblances combinatoires : en effet, ici comme dans le cas précédent, si l'on retrouve comme partenaires sémantiques privilégiés les termes dénonçant l'abus, le mensonge et ceux qui indiquent la nécessité de prévenir ces pratiques, on note également la présence quasi-systématique de termes qui font référence à l'argent, et à l'aspect financier de ce type de stratégie publicitaire : " ... plaquer une image environnementale sur des produits, et la transformer en une source de financement profitable "

"L'écoblanchiment est une pratique commerciale trompeuse..."

" ...quel que soit l'investissement financier des entreprises dans des campagnes de "blanchiment écologique" "

"De plus, ils pratiquent le blanchiment vert, c'est-à-dire qu'ils ont utilisé plus d'argent pour créer leur publicité "verte" que... "

"...ces sociétés, qui font de l'écoblanchiment et s'achètent une image écologique... " 
" ...un moyen de s'acheter une belle image "

"...qui utilise l'environnement à des fins purement mercantiles "

On notera au passage que ce résultat n'est peut-être pas tout à fait étonnant, car bien que les termes " écoblanchiment ", " blanchiment vert " et " blanchiment écologique " soient construits comme des extensions métaphoriques du verbe "blanchir " (nettoyer, laver, rendre propre), leur forme renvoie aussi directement au "blanchiment d'argent ", ce qui accentue encore, sans nul doute, leur connotation péjorative. Le rapprochement avec " blanchiment d'argent " est pourtant trompeur, car la notion de greenwashing ne concerne en aucun cas le blanchiment de fonds illicites.

\subsection{Une préférence sémantique pour les questions d'image et de communication}

La dernière famille de termes, composée de "verdissage " et " verdissement écologique " affiche un profil combinatoire encore différent des variantes étudiées ci-dessus puisqu'elle semble privilégier les associations sémantiques avec les termes évoquant la communication :

"Le verdissement d'image désigne les opérations de communication d'une entreprise "

"...l'influence du "verdissage" (français pour "greenwashing") sur l'image écologique du produit et de l'annonceur"

"...est une action de communication ayant pour but de présenter une image de responsabilité environnementale "

"...une image savamment travaillée à coup de campagnes marketing ? "

"Le verdissement d'image peut se loger dans tous les pans de votre communication " "...ce mot signifie "verdir" ou donner une image écologique à des entreprises... "

Il faut en outre souligner que les résultats extraits du corpus montrent qu'il existe un risque d'ambiguïté sémantique du terme "verdissage ", tantôt utilisé, dans les documents consultés, comme un synonyme possible de " greenwashing ", tantôt utilisé pour décrire les efforts (réels) d'une entreprise pour devenir plus écologique, comme le montrent les exemples ci-dessous, tous issus de documents officiels émanant notamment de la Commission européenne (qui dans l'ensemble de ses écrits, n'utilise d'ailleurs le terme " verdissage " que dans son sens " positif ") :

" ...cette étude est destinée à être utilisée pour supporter (sic) la transition d'un système existant, la Charte méditerranéenne pour l'Entreprise, qui cherche à incorporer un degré de "verdissage" dans son fonctionnement"

"Les mesures politiques identifiées en Syrie tendent à se focaliser sur les entreprises et industries de "verdissage" actuellement en activité... " 
"... on constate un accent évident sur le verdissage par une amélioration de la performance environnementale des entreprises existantes "

On note donc que les équivalents de traduction étudiés dans cet article, bien que renvoyant au même concept, peuvent être considérés comme des facettes légèrement différentes d'un même sens global puisqu'ils affichent des prosodies sémantiques qui ne sont pas complètement identiques : ainsi les termes "écoblanchiment ", " blanchiment vert " et " blanchiment écologique " évoquent plus systématiquement les questions d'argent que les autres, les termes " verdissage " et " verdissement d'image " se rapportent avec une fréquence surprenante aux questions d'image et de communication. Ces termes, bien qu'ils apparaissent dans des types de documents identiques, ne sont visiblement pas complètement interchangeables puisqu'ils évoquent des contenus un peu différents.

L'analyse des préférences sémantiques repérables dans le corpus nous a permis de regrouper les équivalents de traduction possibles de greenwashing en trois familles, mais il convient également, pour affiner cette vision d'ensemble, de repérer les différences éventuelles liées à l'usage de ces termes.

\section{Un usage timide ou confiant? Un usage autonome ou de proximité ?}

La première remarque que nous inspire l'analyse du corpus du point de vue de l'usage est qu'il faut distinguer deux types d'usage, un usage "timide " (nous empruntons ici la terminologie de Quirion, à paraître), et un usage plus confiant des termes à l'étude. L'usage " timide " d'un terme se caractérise par le recours à des marques métalinguistiques dont les plus courantes, dans notre corpus sont les guillemets, l'italique et les formules de distanciation (du type " comme l'appellent ", " comme le nomment ", " qu'on appelle parfois ", etc.), signe que ces termes sont choisis avec une certaine réserve par leurs utilisateurs. Un usage confiant se caractérise par l'utilisation du terme sans ces marques métalinguistiques, signe que le locuteur n'est pas en proie à l'insécurité linguistique.

Ainsi, le terme " greenwashing ", dont la morphologie est typiquement anglophone, semble être utilisé avec précaution par les locuteurs, car il apparaît dans 70 \% des textes du corpus systématiquement accompagné de guillemets, ou d'italiques, ce qui est assez surprenant étant donné sa grande fréquence d'utilisation(4) :

"...grâce à la pression exercée par les ONG et aux efforts des professionnels, mais le greenwashing est toujours là "

(4) L'usage un peu "timide " de ce terme est également contredit par le fait que des formes lexicales dérivées, pour certaines adaptées au français, apparaissent dans le corpus, comme par exemple, le " cyber-greenwashing ", " se laisser greenwasher ", "le champion des greenwasheurs ", etc. 
"Les secteurs les plus sanctionnés pour des faits de "greenwashing" sont l'énergie, l'automobile et les produits de grande consommation (alimentation, lessive...) "

C'est également le cas pour " badigeonnage vert ", " blanchiment écologique ", et " verdissage ", qui sont utilisés plus fréquemment avec des marques métalinguistiques indiquant une insécurité linguistique que sans :

"Ce "blanchiment écologique" minimise et banalise la nécessité impérative de changer les comportements de consommation. "

"Les écologistes critiquent le maire Fabrice Roussel, lui reprochant, entre autre, un "verdissage" (c'est le phénomène du "greenwashing") de sa communication... "

"Ainsi le "greenwashing" ou "badigeonnage vert" désigne une " communication qui utilise de façon abusive..."

En revanche, les termes " maquillage vert ", " désinformation verte ", " blanchiment vert ", " verdissement d'image ", " mascarade écologique " et " écoblanchiment " semblent faire l'objet d'un usage plus confiant, puisqu'ils apparaissent plus souvent sans guillemets qu'avec :

"...ce n'est pas de l'innovation, mais du blanchiment vert de l'élevage industriel... "

"On peut se demander si ce n'est pas ici de la désinformation verte "

"... tous pratiquent l'écoblanchiment afin de rassurer les consommateurs sur... "

"...fait référence au verdissement d'image d'une organisation "

"Le bon éco-étiquetage contribue à prévenir (mais n'élimine pas) la mascarade écologique. " "C'est ce que l'on appelle du maquillage vert. "

Enfin, un dernier constat : deux des termes étudiés (" badigeonnage vert " et " verdissage "), ne sont que rarement utilisés de façon autonome et ne sont souvent introduits dans une phrase que lorsqu'ils sont accolés au terme " greenwashing " (le suivant ou le précédant, parfois entre parenthèses), signe que leur usage n'est qu'un usage dépendant, de proximité (ils sont alors visiblement utilisés dans ce cas pour "franciser " ou reformuler le terme " greenwashing ") :

"Parmi toutes les allégations environnementales qui fleurissent un peu partout, avec des risques de greenwashing ("badigeonnage vert")..."

"En français "verdissage", le greenwashing est une stratégie de marketing et de publicité qui repose..." "

La courte analyse présentée ci-dessus n'est en aucun cas exhaustive, et pourrait encore être enrichie d'autres pistes de réflexion ; elle n'a pas pour objectif, comme nous l'avons dit en introduction, de faire des recommandations sur la meilleure traduction en français du terme greenwashing, mais seulement d'apporter des éléments de réflexion préalables à la traduction. 
On en retire quand même qu'opter systématiquement pour la standardisation terminologique à l'intérieur d'un texte ou d'un groupe de textes peut ne pas être un bon réflexe de traduction, surtout lorsque de nombreuses variantes, y compris officielles, coexistent et qu'il s'agit d'un domaine à la mode et populaire, comme peut l'être celui du développement durable. En fonction du contenu du texte à traduire, l'une ou l'autre variante, si l'on tient compte de ses préférences sémantiques notamment, conviendra peut-être mieux.

Sur le plan terminologique, on remarque également que la popularisation d'un terme à la mode comme greenwashing peut provoquer, par " effet rebond ", l'apparition d'autres termes qui lui sont liés, par la forme et par le sens (on trouve ainsi dans notre corpus mention des termes " sciencewashing " et du " ethic-washing "(5)), complexifiant ainsi la tâche du traducteur.

Pascaline.dury@univ-lyon2.fr

\section{Références bibliographiques}

Dictionnaires Oxford en ligne : http://www.oxforddictionaries.com/, dernière consultation le 30/10/2013.

LOUW Bill, 1993, "Irony in the text or insincerity in the writer? The diagnostic potential of semantic prosodies", in BAKER, Mona, FRANCIS, Gill et Elena TOGNINI-BONELLI, (éd.), Text and Technology: In Honour of John Sinclair. Amsterdam, John Benjamins, pp. 157-176.

PARTINGTON, Alan, 2004, "Utterly content in each other's company : semantic prosody and semantic preference", in International Journal of Corpus Linguistics 9-1, pp. 31-156.

QUIRION, Jean, à paraître, "Pour une néologie sociale ", in DURY Pascaline et al., (éd.), La néologie en langue de spécialité : détection, implantation et circulation des nouveaux termes, Lyon, Presses Universitaires de Lyon, pp. 88-105.

SINCLAIR, John McHardy, 1987, Looking up, London, Collins.

STUBBS, Michael, 2001, Words and Phrases, Oxford, Blackwell.

Pascaline Dury est Maitre de Conférences en terminologie et traduction à l'Université Lumière Lyon 2, et fait partie du CRTT (Centre de Recherche en Terminologie et Traduction). Elle s'intéresse à la terminologie médicale et à la terminologie de l'environnement, notamment d'un point de vue diachronique.

(5) On trouve par exemple dans le corpus : " ...les entreprises ou les organisations peuvent très bien pratiquer "l'éthic-washing" (mascarade éthique). Cela équivaut au greenwashing, sauf que les promesses infondées ou sans pertinence concernent cette fois-ci les questions sociales plus qu'écologiques " ou bien encore : " le sciencewashing consiste à faire passer pour scientifique une pratique qui ne l'est pas afin de servir des intérêts privés et influencer l'opinion publique ". 